\title{
Reductions in hypothalamic Gfap expression, glial cells and a-tanycytes in lean and hypermetabolic Gnasxl-deficient mice
}

Andrew P. Holmes ${ }^{1}$, Shi Quan Wong ${ }^{2}$, Michela Pulix², Kirsty Johnson², Niamh S. Horton², Patricia Thomas², João Pedro de Magalhães ${ }^{1 *}$ and Antonius Plagge ${ }^{2^{*}}$

\begin{abstract}
Background: Neuronal and glial differentiation in the murine hypothalamus is not complete at birth, but continues over the first two weeks postnatally. Nutritional status and Leptin deficiency can influence the maturation of neuronal projections and glial patterns, and hypothalamic gliosis occurs in mouse models of obesity. Gnasxl constitutes an alternative transcript of the genomically imprinted Gnas locus and encodes a variant of the signalling protein $\mathrm{Ga}_{\mathrm{st}}$ termed $X \mathrm{La}_{\mathrm{s}}$ which is expressed in defined areas of the hypothalamus. Gnasxl-deficient mice show postnatal growth retardation and undernutrition, while surviving adults remain lean and hypermetabolic with increased sympathetic nervous system (SNS) activity. Effects of this knock-out on the hypothalamic neural network have not yet been investigated.
\end{abstract}

Results: RNAseq analysis for gene expression changes in hypothalami of Gnasxl-deficient mice indicated Glial fibrillary acid protein (Gfap) expression to be significantly down-regulated in adult samples. Histological analysis confirmed a reduction in Gfap-positive glial cell numbers specifically in the hypothalamus. This reduction was observed in adult tissue samples, whereas no difference was found in hypothalami of postnatal stages, indicating an adaptation in adult Gnasxl-deficient mice to their earlier growth phenotype and hypermetabolism. Especially noticeable was a loss of many Gfap-positive a-tanycytes and their processes, which form part of the ependymal layer that lines the medial and dorsal regions of the $3^{\text {rd }}$ ventricle, while $\beta$-tanycytes along the median eminence (ME) and infundibular recesses appeared unaffected. This was accompanied by local reductions in Vimentin and Nestin expression. Hypothalamic RNA levels of glial solute transporters were unchanged, indicating a potential compensatory upregulation in the remaining astrocytes and tanycytes.

Conclusion: Gnasxl deficiency does not directly affect glial development in the hypothalamus, since it is expressed in neurons, and Gfap-positive astrocytes and tanycytes appear normal during early postnatal stages. The loss of Gfap-expressing cells in adult hypothalami appears to be a consequence of the postnatal undernutrition, hypoglycaemia and continued hypermetabolism and leanness of Gnasxl-deficient mice, which contrasts with gliosis observed in obese mouse models. Since a-tanycytes also function as adult neural progenitor cells, these findings might indicate further developmental abnormalities in hypothalamic formations of Gnasxl-deficient mice, potentially including neuronal composition and projections.

Keywords: Gnas, Genomic imprinting, Hypothalamus, Tanycyte, Gfap, Glia, Energy homoeostasis, RNAseq

\footnotetext{
* Correspondence: jp@senescence.info; a.plagge@liverpool.ac.uk

${ }^{1}$ Integrative Genomics of Ageing Group, Institute of Integrative Biology,

University of Liverpool, Crown Str, Liverpool L69 7ZB, UK

${ }^{2}$ Cellular and Molecular Physiology, Institute of Translational Medicine,

University of Liverpool, Crown Str, Liverpool L69 3BX, UK
} 


\section{Background}

The hypothalamus exerts important regulatory functions in whole-body energy homeostasis, including food intake, energy expenditure and SNS activity [1-3]. A neuronal network between several hypothalamic nuclei, including the arcuate nucleus (Arc), dorsomedial nucleus (DMH) and paraventricular nucleus $(\mathrm{PVH})$, integrates peripheral hormonal signals like leptin, insulin and ghrelin, which reflect levels of energy reserves and feeding status. The pathways of leptin action, to inhibit food intake and stimulate energy expenditure when adipose tissue fat reserves are high, have been elucidated in some detail. Leptin inhibits orexigenic neuropeptide Y (NPY) neurons and activates anorexigenic pro-opiomelanocortin (POMC) neurons in the arcuate nucleus [3]. This leads to activation of the melanocortin system (neurons expressing MC4 and MC3 receptors) in the PVH, parabrachial nucleus, dorsal vagal complex and the intermediolateral cell column of the spinal cord. While MC4R-expressing neurons in the $\mathrm{PVH}$ and parabrachial nucleus mainly regulate food intake, those in other hypothalamic nuclei, the dorsal vagal complex and the spinal cord have been implicated in the control of energy expenditure and SNS activity [1-3].

In rodents, the formation of the hypothalamic neural network is incomplete at birth as, for example, axonal projections from the Arc to the PVH and DMH develop and mature over the first two weeks postnatally [4-6]. Leptin has an important neurotrophic function during this postnatal period. Deficiency of leptin results in diminished POMC and NPY/Agrp projections from the Arc [4]. Full functionality of the neuronal circuits controlling food intake and energy expenditure is established by weaning age. Apart from leptin deficiency, nutritional status at embryonic or postnatal stages can also impact on the formation of neural circuits controlling energy homeostasis [5, 7]. Perturbations during these developmental stages do not always lead to obesity as, for example, postnatal undernutrition results in permanent leanness, transformation of white to brown-like adipose tissue, increased hypothalamic leptin and melanocortin sensitivity and increased leptin receptor expression [8-10]. The changes in molecular mechanisms and neural networks leading to such permanent leanness have not yet been investigated in detail.

Apart from neuronal networks, glial cells and tanycytes, which are located within the ependymal layer of the $3^{\text {rd }}$ ventricle, also participate in the hypothalamic regulation of energy homeostasis and are responsive to metabolic status [11-14]. Both cell types form protrusions that are in contact with blood capillaries, or the ventricular surface in the case of tanycytes, and neurons, thus enabling the transport and exchange of nutrients, neurotransmitters and hormones. A responsiveness of these non-neuronal cells to whole-body energy status is indicated by data showing hypothalamic gliosis and increased Gfap expression in genetically or diet-induced obese models [15-20]. Furthermore, recent work has highlighted a role for tanycytes as neural progenitor cells that contribute to the hypothalamic energy homeostasis regulating network [21-24]. Different subsets of tanycytes show a neurogenic response to specific signalling factors as well as to a high-fat diet [24-28].

The Gnas locus, which encodes variants of the Gprotein $\alpha$-stimulatory subunit $\left(G \alpha_{s}\right)$, is involved in the hypothalamic regulation of energy balance [29]. The locus consist of a complex arrangement of proteincoding and non-coding transcripts, which are expressed from alternative promoters (Fig. 1) [30, 31]. In addition to the well-known $\mathrm{G} \alpha_{\mathrm{s}}$ protein, an $\mathrm{NH}_{2}$-terminal variant (extra-large $\alpha_{\mathrm{s}} ; \mathrm{XL} \alpha_{\mathrm{s}}$ ) is expressed from a separate promoter and transcript $($ Gnasxl). Both proteins stimulate cAMP production after activation of seven-transmembrane receptors, but $\mathrm{XL} \alpha_{\mathrm{s}}$ can also exert a $\mathrm{G} \alpha_{\mathrm{q} / 11}$-like function and increase inositol 1,4,5-trisphosphate signalling in some cell types [32, 33]. The Gnas locus is epigenetically regulated by a mechanism termed genomic imprinting, which results in monoallelic gene expression according to parental origin [30]. DNA methylation acquired in the female germline silences the expression of Gnasxl on the maternally inherited allele. By contrast, Gnas remains biallelically expressed in most tissues of the offspring, apart from a few cell types, including specific brain regions, in which the paternally inherited Gnas allele is silenced (Fig. 1) [29-31].

Due to the monoallelic expression of these transcripts, heterozygous knock-out (KO) mutations were used to analyse the roles of Gnas and Gnasxl in energy homeostasis, which revealed opposite phenotypes [29, 34]. Brain-

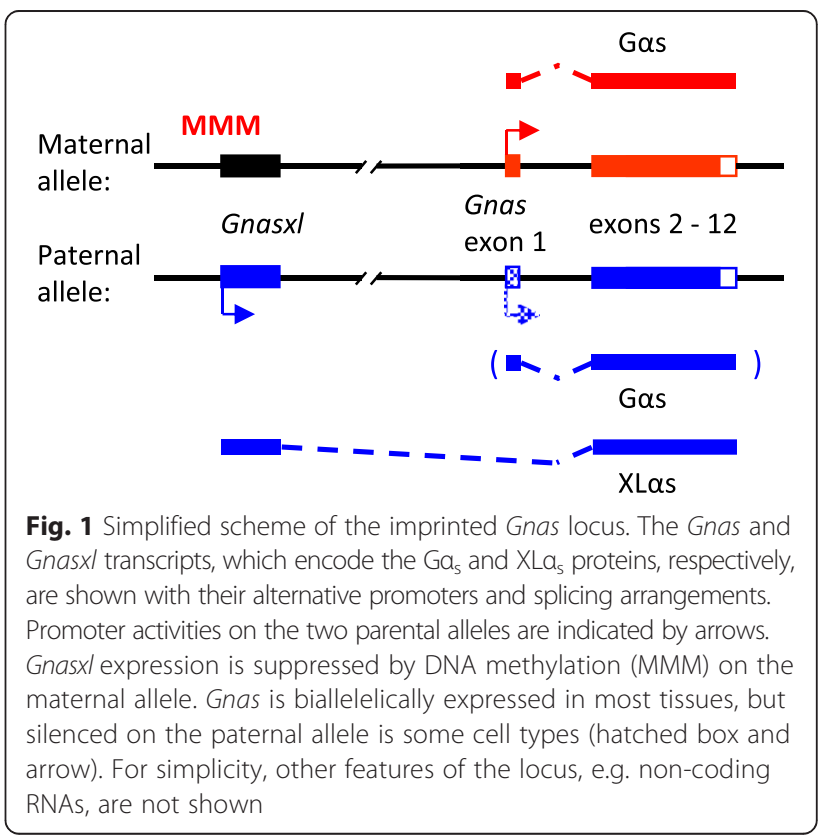


specific knock-out of Gnas expression from the maternal allele results in obesity due to disrupted melanocortin receptor $/ \mathrm{G \alpha}$ s signalling and consequently reduced energy expenditure [35, 36]. By contrast, deletion of Gnasxl expression (from the paternal allele, Gnas $x l^{\mathrm{m}+/ \mathrm{p}-}$ ) causes a lean and hypermetabolic phenotype associated with increased energy expenditure and elevated SNS activity [37-39]. This phenotype of adult KO mice follows an impaired postnatal development, which is characterised by growth retardation, reduced feeding, hypoglycaemia and lack of adipose reserves [38]. $\mathrm{XL} \alpha_{\mathrm{s}}$ is expressed in defined regions of the brain at neonatal and adult stages; its neonatal expression in some peripheral tissues is, however, down-regulated towards weaning [32, 38-40]. Within the hypothalamus, Gnasxl is present in neurons of the Arc, $\mathrm{DMH}, \mathrm{PVH}$, lateral hypothalamus and the suprachiasmatic nucleus (SCN) [40]. It is currently unclear how Gnasxl functions within the neural energy homeostasis network, which receptor(s) might signal via $X L \alpha_{s}$ in the brain, and whether the postnatal development of the hypothalamic network might be impaired as a consequence of the neonatal undernutrition experienced by Gnas $x l^{\mathrm{m}+/ \mathrm{p}-}$ pups.

Whole transcriptome sequencing (i.e., RNAseq) has been shown to have many advantages for gene expression profiling when compared to microarrays [41], including in the context of the rodent brain [42]. Taking an unbiased approach to analyse gene expression changes in hypothalami of Gnasxl-deficient mice via RNAseq, we identified a 2-fold down-regulation of the glial marker Gfap. This unexpected finding was confirmed by histological analyses of adult hypothalami, which showed reduced Gfap-positive cell numbers, while initial glial development at neonatal stages was not different. The loss of glial cells in adult hypothalami appears to be a consequence associated with the lean Gnasxl-deficient phenotype, and our observations complement opposite findings of gliosis in obese mouse models. Furthermore, we show not only astrocytes, but also Gfap-expressing $\alpha$-tanycytes to be diminished. Since $\alpha$-tanycytes have a role as neural progenitor cells, our data point towards potentially more far-reaching impacts on the development or maintenance of the hypothalamic energy homeostasis network.

\section{Results}

Transcriptome analysis indicates reduced Gfap expression in hypothalami of Gnasxl-deficient mice

To take an unbiased approach to screen for changes in gene expression in hypothalami of lean and hypermetabolic Gnasx $l^{\mathrm{m}+/ \mathrm{p}-}$ mice, we undertook a RNAseq transcriptome analysis using the Illumina platform. Hypothalamus RNA

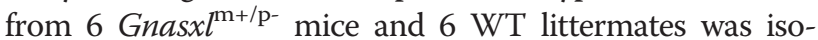
lated and two samples of identical genotype were combined to obtain a total of 3 Gnasx $l^{\mathrm{m}+\mathrm{p}-}$ and 3 WT RNA sample pools for library preparations and sequencing. The six resulting sequencing files were mapped individually using TopHat 2.0.4 to the mm10 build of the mouse genome. Using the Cufflinks package 2.0.2, we identified a total of 332 significantly differentially expressed genes (Additional file 1; Gene Expression Omnibus (GEO) accession number GSE75878). Most of these changes were within the range from 4-fold increase to 4-fold decrease, although some small nucleolar RNAs showed particularly high fold-changes (Additional file 1). Notably Gnasxl RNA, which is lost in the $\mathrm{KO}$ mice [38], was not identified as differentially expressed. This is most likely due to the complex structure of the Gnas locus with its transcript-specific alternative promoters and mostly shared exons [30, 31], which are difficult to resolve. To analyse the dataset of differentially expressed genes further for association with common processes and pathways [41], a functional enrichment analysis was undertaken and revealed statistically significant enriched categories amongst overexpressed genes related to two broad categories: ribosomes, rRNA binding and translation; and mitochondria and electron transport chain (Additional file 2). No statistically significant categories were found amongst down-regulated genes after correcting for multiple hypotheses testing (Additional file 2).

From the RNAseq list of genes (Additional file 1), we selected a subset, which have been implicated in the literature with hypothalamic regulation of energy homeostasis, for further verification by qRT-PCR. The candidate gene Glial fibrillary acidic protein (Gfap) showed a similar 2-fold down-regulation by RNAseq and qRT-PCR (Gnasx $^{\mathrm{m}+/ \mathrm{p}-}$ RNAseq fold change: $0.48 \pm 0.04$ SEM, $p<$ 0.0001, $n=3$ per genotype; qRT-PCR fold change: $0.46 \pm$ 0.18 SEM; $p<0.05, n=3$ per genotype) (Fig. 2). Since this down-regulation of hypothalamic Gfap RNA in lean and hypermetabolic Gnasx $l^{\mathrm{m}+\mathrm{p}-}$ mice contrasts with the up-regulation and gliosis found in obese mouse models [15-20], we decided to further investigate Gfap protein expression on the histological level.

\section{Reduced glial cell numbers in adult, but not postnatal, hypothalami of Gnasx $I^{\mathrm{m}+/ \mathrm{p}-}$ mice}

To assess the localisation and number of hypothalamic Gfap-positive cells, immunohistochemistry (IHC) was carried out on brain sections from adult Gnasxl WT and KO littermates. Apart from astrocytes, Gfap was found to be expressed in a subset of periventricular tanycytes and their processes, which extend from the surface of the $3^{\text {rd }}$ ventricle into the hypothalamic parenchyma (Fig. 3, Fig. 5 and Fig. 6) [14]. Only the $\alpha 1$ and $\alpha 2$ subpopulations, which are located along the dorsal ventricle and the Arc, were found to express Gfap [14, 27, 28]. The $\beta 1$ and $\beta 2$ tanycytes, which line the ventral ventricle along the infundibular recesses and ME, respectively, were negative for the glial marker. In Gnasx $l^{\mathrm{m}+/ \mathrm{p}-}$ samples, we found fewer Gfap-positive cells in all areas 


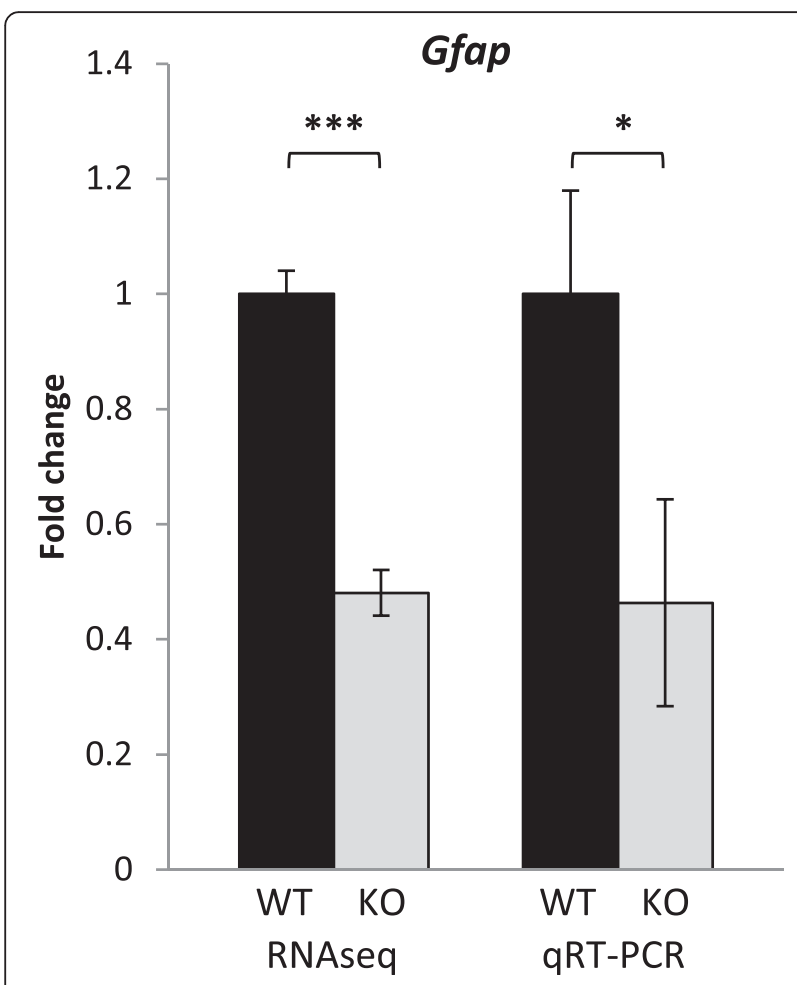

Fig. 2 Reduced Gfap RNA expression levels in Gnasx ${ }^{\text {m+/p- }}$ hypothalami. A similar 2-fold down-regulation of Gfap RNA levels was found by RNAseq and qRT-PCR in Gnasxl knock-out hypothalami $\left({ }^{* * *} p<0.0001\right.$; ${ }^{*} p<0.05$; Student's independent $t$-test; $n=3 \mathrm{WT}$ and $3 \mathrm{KO}$ pools of RNA with each pool consisting of two individual samples of the same genotype)

along the rostro-caudal and dorso-ventral axes of the hypothalamus, including the $\mathrm{PVH}, \mathrm{DMH}, \mathrm{Arc}, \mathrm{ME}$ and PH (Fig. 3a-f). Quantification indicated a $41 \%$ reduction in Gfap-positive cell numbers in posterior areas of the hypothalamus $(\mathrm{WT}=305 \pm 21$ vs $\mathrm{KO}=179 \pm 23$ cells/section \pm sem, ${ }^{* * * * *} p<0.001, t$-test, $n=11$ matched pairs of sections from $5 \mathrm{WT}$ and $5 \mathrm{KO}$ mice), while anterior and central regions showed $26 \%$ fewer glial cells $(\mathrm{WT}=1110 \pm 42$ vs $\mathrm{KO}=937 \pm 35$ cells/section \pm sem, *** $p<0.01, t$-test, $n=11$ matched pairs of sections from 5 WT and $5 \mathrm{KO}$ mice) (Fig. 3g). In the anterior hypothalamus, the suprachiasmatic nucleus (SCN) also contained fewer Gfap-positive astrocytes (Fig. 4). To investigate whether a reduced number of Gfap-expressing cells is specific to the hypothalamus or occurs generally throughout the brain, we additionally assessed cell numbers in the hippocampus. We found no significant difference in this brain region $(\mathrm{WT}=769 \pm 16$ vs $\mathrm{KO}=746 \pm 19$ cells $/ \mathrm{sec}$ tion \pm sem, $p>0.05$, $t$-test, $n=15$ matched pairs of sections from $5 \mathrm{WT}$ and $5 \mathrm{KO}$ mice) (Additional file 3). These findings indicate that a reduction in Gfap expression and glial cell numbers is a characteristic feature of the hypothalamus area of Gnasx $^{\mathrm{m+}+\mathrm{p}^{-}}$brains.
Since Gnasxl is expressed in hypothalamic neurons, but not in glia or the ependymal layer of the hypothalamus (Additional file 4) [40], the reduction in Gfapexpressing cells in the adult $\mathrm{KO}$ samples appears to be a consequence of Gnasxl deficiency and an adaptation to the severe postnatal undernutrition phenotype of

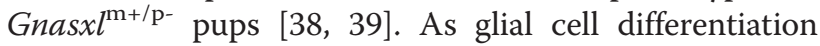
follows neuronal differentiation and mainly occurs at late embryonic and early postnatal stages [14, 43], we investigated whether this reduction in hypothalamic Gfap-positive cells has a postnatal onset. Histological comparison of Gfap expression in hypothalami of postnatal day 15 (P15) samples did not, however, show a significant difference in cell numbers between the two genotypes (Fig. 5) (WT $=382 \pm 25$ vs $\mathrm{KO}=440 \pm 34$ cells/section \pm sem, n.s. $p>0.05, t$-test, $n=33$ matched pairs of sections from $3 \mathrm{WT}$ and $3 \mathrm{KO}$ ). Earlier postnatal stages (P1, P5 and P10) showed overall lower numbers of Gfap-positive cells, but no difference was found between WT and KO samples (data not shown). These findings indicate that deficiency of Gnasxl does not impact on the initial formation and differentiation of glial cells, but that their reduction in adult hypothalami is a consequence of the KO phenotype, which includes postnatal growth retardation and undernutrition (hypoglycaemia, hypolipidemia) as well as increased metabolic rate driven by elevated SNS activity during adult stages [37-39].

\section{Loss of a-tanycytes and their processes}

From the Gfap-IHC (Fig. 3) it appears that not only astrocytes are affected, but also the $\alpha$-subset of tanycytes. $\alpha$-tanycytes facilitate the exchange of nutrients and signalling molecules through their long processes, which are in contact with the ventricular surface and bridge to neurons and blood vessels far into the hypothalamic parenchyma [14]. Furthermore, recent reports have associated $\alpha$-tanycytes with adult neural progenitor cell functions [14, 24, 27, 28]. To further investigate the effect of Gnasxl-deficiency on this cell type, we undertook IHC for the intermediate filament Vimentin, which is a marker for all tanycytes as well as ependymocytes [27]. We found a strong reduction in Vimentin-positive tanycyte processes specifically in the $\alpha$-subset of tanycytes, while the $\beta$-subpopulation appeared normal (Fig. 6). Lack of Gfap and Vimentin staining largely coincided in the Gnasxl $l^{\mathrm{m+} / \mathrm{p}-}$ samples, which points to a loss of many of the double-positive $\alpha$-tanycyte processes after postnatal day 15 and is in accordance with the reduced number of Gfap-positive cell bodies (Fig. 3). Remaining Vimentin-positive cells without processes located in the ependymal layer of the $\alpha$-tanycyte region most likely represent ependymocytes [27]. In line with these findings, a trend towards reduced vimentin gene expression, although not significant, was observed in 

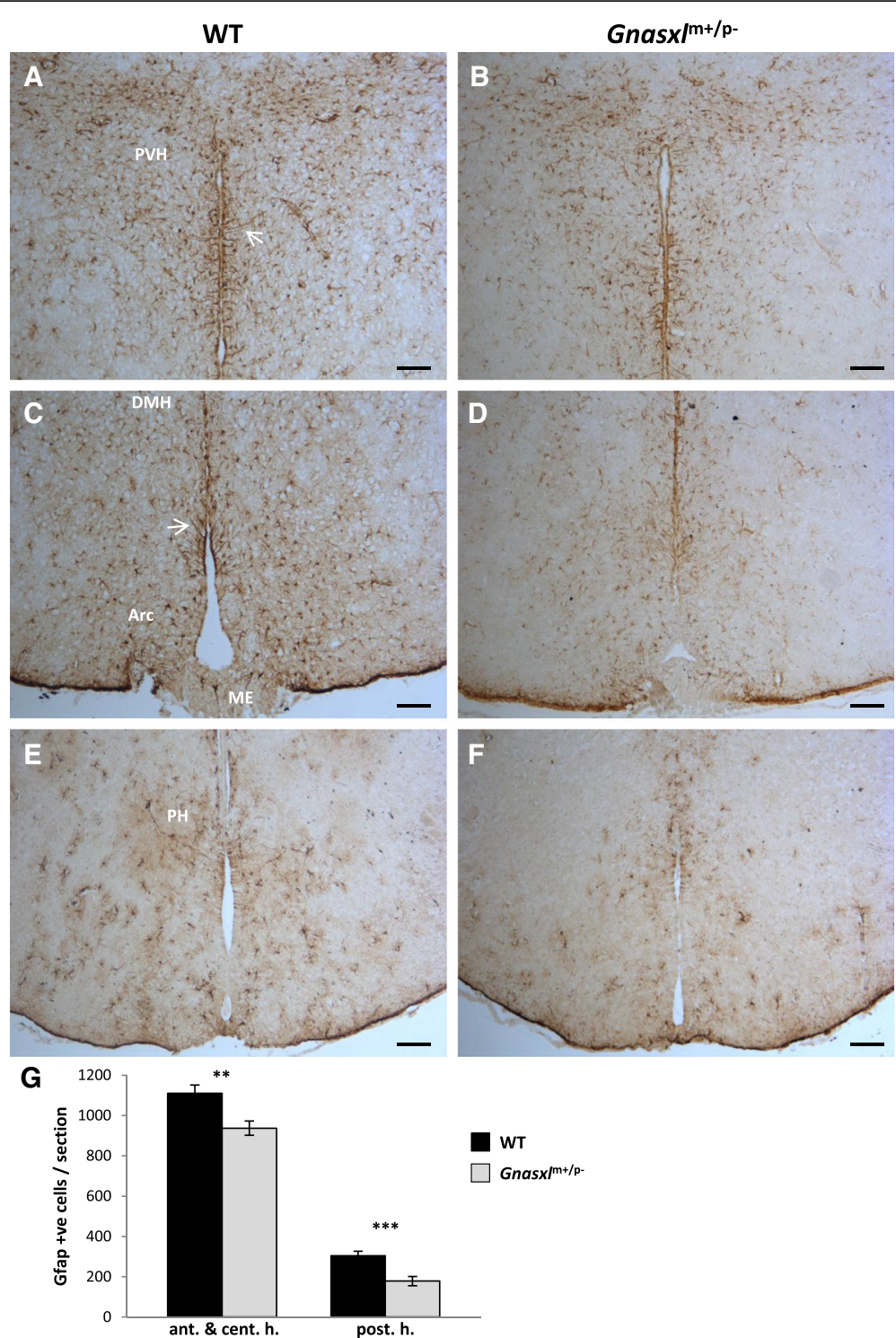

Fig. 3 Lower Gfap-positive cell counts in hypothalami of adult Gnasx ${ }^{\mathrm{m}+/ \mathrm{p}-}$ mice. IHC for Gfap on coronal brain sections from WT (a, c, e) and KO $(\mathbf{b}, \mathbf{d}, \mathbf{f})$ littermates at different rostro-caudal levels. In addition to astrocytes, the a-subset of ependymal tanycytes and their processes (white arrows) were found to express Gfap. a and $\mathbf{b}$ Anterior-dorsal hypothalamus at the level of the Paraventricular Nucleus (PVH). $\mathbf{c}$ and $\mathbf{d}$ Central-ventral hypothalamus at the level of the Arcuate Nucleus (Arc). e and $\mathbf{f}$ Level of the posterior hypothalamus (PH). Scale bar $=100 \mu \mathrm{m}$. $\mathbf{g}$ Quantification of Gfap-expressing cells at the anterior-central and posterior levels, respectively, (anterior \& central: WT $=1110 \pm 42$ vs KO $=937 \pm 35$ cells/section \pm sem, ${ }^{*} p<0.01$, t-test, $n=11$ matched pairs of sections from $5 \mathrm{WT}$ and $5 \mathrm{KO}$ mice; posterior: $\mathrm{WT}=305 \pm 21$ vs $\mathrm{KO}=179 \pm 23$ cells $/ \mathrm{section} \pm$ sem, *** $p<0.001$, $t$-test, $n=11$ matched pairs of sections from $5 \mathrm{WT}$ and $5 \mathrm{KO}$ mice)

the RNAseq analysis of whole hypothalamus lysates (Additional file 1). Additional investigations into the expression pattern of the neural progenitor cell marker Nestin showed a localised reduction of the protein in the region of $\alpha$-tanycytes (Fig. 7), similar to the changes observed for Vimentin. Whether these changes in the $\alpha$-tanycyte population impact on neural progenitor cell functions $[14,24,27,28]$ and hypothalamic network maintenance in Gnasxl $l^{\mathrm{m}+/ \mathrm{p}-}$ mice remains to be determined.
Astrocytes and tanycytes express specific solute transporter proteins for glucose (Slc2a1/Glut1) and excitatory amino acids (Slc1a2/Glt1 and Slc1a3/Glast1), thus regulating their availability and exchange between compartments of the hypothalamic microenvironment $[14,44,45]$. Despite the cellular changes described above we did, however, not detect a reduction in expression levels of these transporter genes in whole hypothalamus lysates of Gnasx $l^{\mathrm{m}+/ \mathrm{p}-}$ mice by RNAseq analysis or qRT-PCR (Additional files 1 and 5), which 


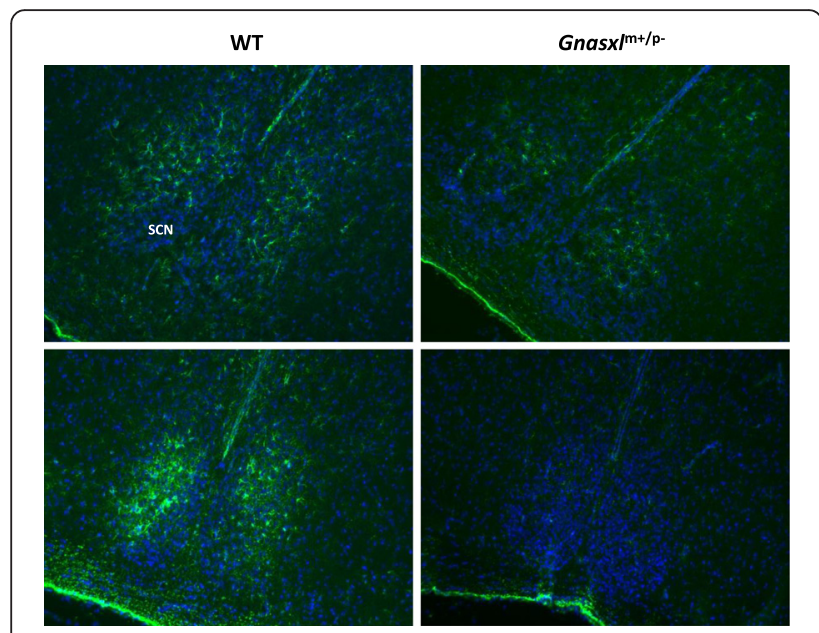

Fig. 4 Fewer Gfap-expressing astrocytes in the suprachiasmatic nucleus (SCN). Gfap-immunofluorescence (green) in the SCN of two adult WT and Gnasx ${ }^{m+/ p-}$ littermates (nuclear stain: DAPI). Images taken at original magnification $100 x$ might be due to compensatory up-regulation in the remaining glial cell population.

\section{Discussion}

The unbiased assessment of gene expression changes in hypothalami of adult Gnasxl-deficient mice via RNAseq revealed a 2-fold down-regulation of the glial marker Gfap, and further histological examination indicated reduced numbers of Gfap-positive astrocytes and $\alpha$ tanycytes. These findings were unexpected, since Gnasxl is not expressed in glial cells or in the ependymal layer of the $3^{\text {rd }}$ ventricle, but is present in neurons of the Arc, $\mathrm{DMH}, \mathrm{PVH}$, lateral hypothalamus and the SCN [40]. Furthermore, Gfap-positive cell numbers were not diminished at postnatal stages, when the developmental process of glia and tanycyte differentiation reaches completion in mice [14, 43, 46, 47]. This indicates that the initial formation of these cell types is not impaired by lack of $X L \alpha_{s}$, but that their reduction in adult hypothalami must be a consequence of the phenotype of the
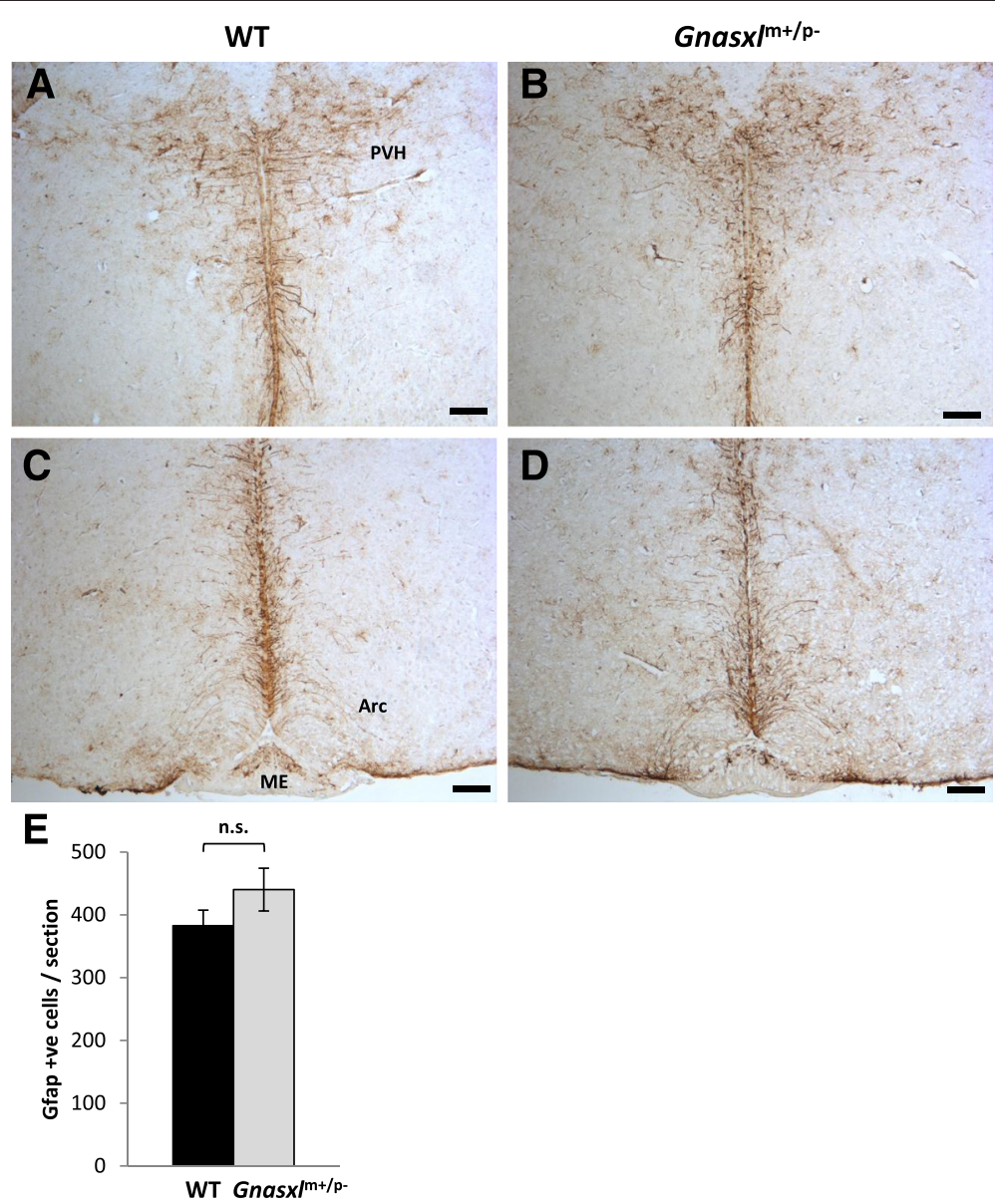

Fig. 5 Glial cell numbers are not different in hypothalami of postnatal Gnasx $\left.\right|^{m+/ p^{-}}$mice. Gfap IHC on postnatal day 15 brain sections from WT ( $\mathbf{a}$ and $\mathbf{c}$ ) and $\mathrm{KO}$ (b and $\mathbf{d}$ ) littermates. $\mathbf{a}$ and $\mathbf{b}$ Anterior-dorsal hypothalamus at the level of the $\mathrm{PVH}$; (c and $\mathbf{d}$ ) central-ventral hypothalamus at the level of the Arc. Scale bar $=100 \mu \mathrm{m}$. e Quantification of Gfap-positive cells: WT $=382 \pm 25 \mathrm{vs} \mathrm{KO}=440 \pm 34$ cells/section \pm sem, $p>0.05, t$-test, $n=33$ matched pairs of sections along the rostro-caudal axis of the hypothalami of $3 \mathrm{WT}$ and $3 \mathrm{KO}$ mice 


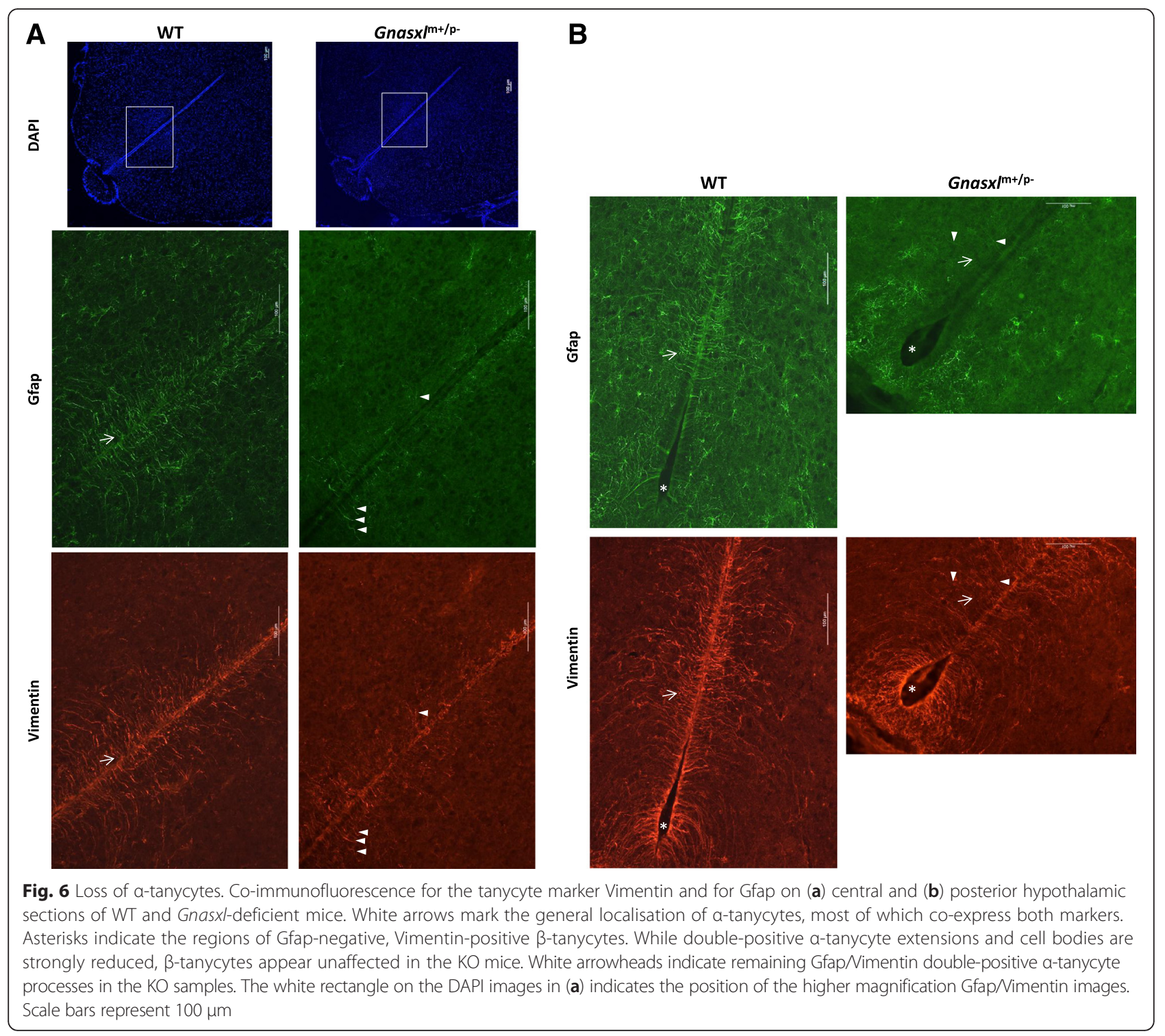

Gnasxl KO mice, which comprises post-natal undernutrition, continued hypoglycaemia, reduced body weight and fat mass as well as hypermetabolism [38, 39].

It is remarkable in this context that nutritional programming in rats, i.e. postnatal under-nutrition, results in adult offspring with a very similar phenotype to Gnasxl-deficient mice, including reduced body weight and fat mass, transformation of white adipose tissue to metabolically stimulated 'brown-like' adipose tissue and hypoleptinemia $[8-10,38,39]$. However, changes in hypothalamic neuronal networks have been little analysed at the histological level in rodent models of postnatal undernutrition [48], and glial or tanycyte abnormalities have not been reported so far. In support of our findings in Gnasxl $^{\mathrm{m}+/ \mathrm{p}^{-}}$mice, some studies on the effects of foetal and/or postnatal protein restriction and malnutrition described similar reductions in Gfap expression and glial cell numbers in the suprachiasmatic nucleus and the medial preoptic area $[49,50]$. By contrast, genetic or dietinduced mouse models of obesity have been much better characterised for changes in the hypothalamus. Specifically, observations of gliosis and increased Gfap expression in obese mice contrast with the glial effects we identified in lean Gnasxl KOs [15-20]. The gliosis observed in obese mice leads to increased glial ensheathment and reduced numbers of synapses on POMC and NPY neurons in the Arc [18]. Whether, correspondingly, glial processes surrounding hypothalamic neurons in Gnasxl-deficient mice are reduced remains to be determined on an ultrastructural level. Any consequences of such glial changes on the activity of the neural network regulating energy homeostasis are only beginning to be explored, although in obese mice a reduced inhibitory synaptic input on POMC neurons was detected $[17,18]$. 

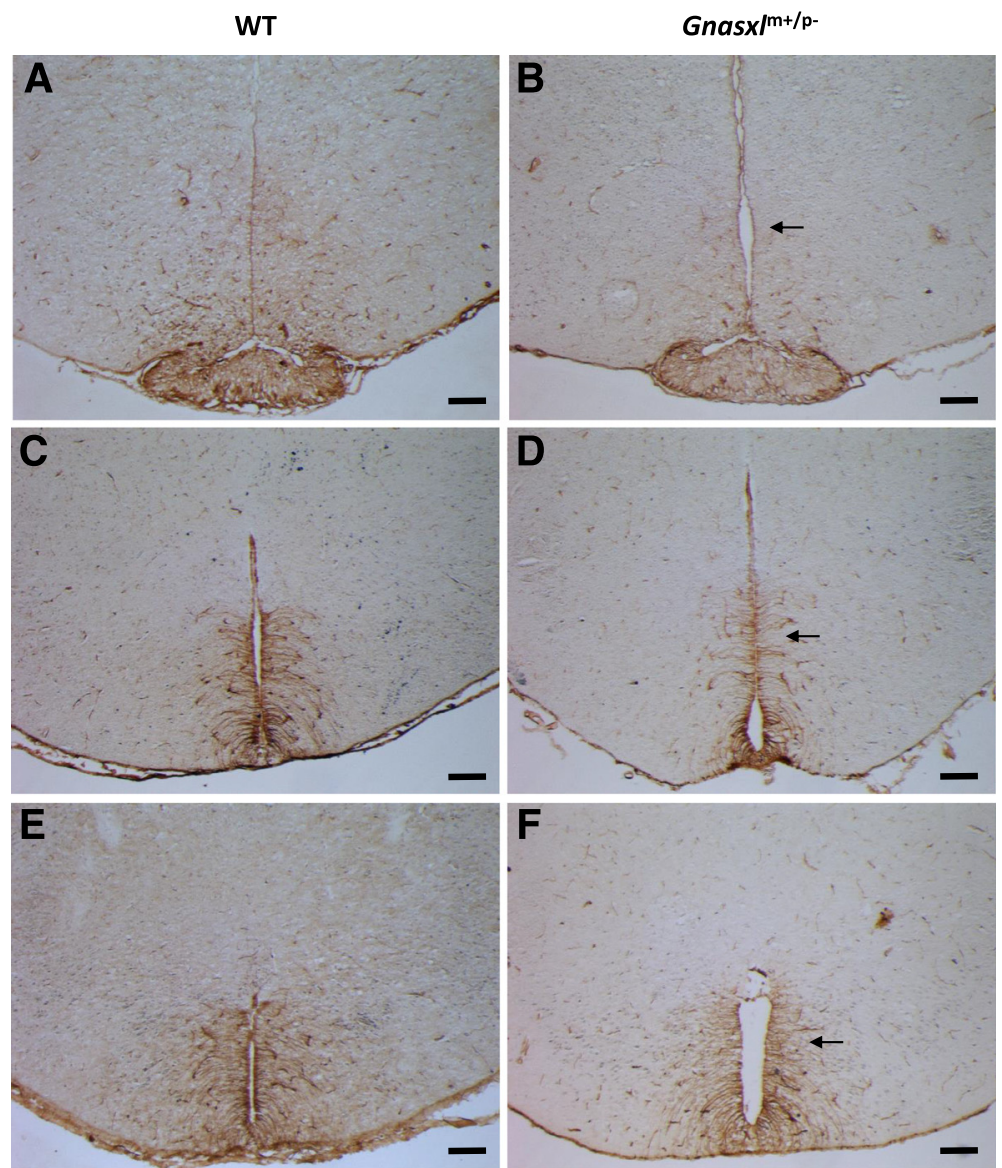

Fig. 7 Reduced expression of Nestin in a-tanycyte region. Immunohistochemistry for the neural progenitor cell marker Nestin shows reduced expression in the region of a-tanycytes (arrows), but no difference in the more ventrally located $\beta$-tanycyte population. Pairs of images from three different WT and Gnasx $^{\mathrm{m+} / \mathrm{p}-}$ adult littermates are shown for medial $(\mathbf{a}, \mathbf{b})$ to posterior $(\mathbf{c}, \mathbf{d}, \mathbf{e}, \mathbf{f})$ positions of the hypothalamus. Scale bars indicate $100 \mu \mathrm{m}$

A loss of Gfap expression in Gnasxl KO mice was especially evident in the $\alpha$-subset of tanycytes, which are part of the ependymal layer lining the $3^{\text {rd }}$ ventricle along the Arc, ventromedial hypothalamus and DMH [14]. The $\beta$-subset of tanycytes, which is located more ventrally along the infundibular recesses of the $3^{\text {rd }}$ ventricle and the ME, does not express Gfap, but both subsets are marked by expression of Vimentin [13, 14, 27, 28]. While Vimentin IHC of $\beta$-tanycytes and their processes appeared undiminished in Gnasx $l^{\mathrm{m}+/ \mathrm{p}^{-}}$mice, it was reduced in a similar pattern to Gfap in the $\alpha$-tanycyte subset. Few double-positive $\alpha$-tanycyte processes remained. Some Vimentin-positive/Gfap-negative cells without processes were still detectable in the ependymal layer of the $\alpha$-tanycyte region of Gnasxl KOs, which most likely represent Vimentin-expressing ependymocytes [27]. Although we also detected a trend towards reduced vimentin gene expression in the RNAseq experiment, this was not significant in the context of lysates from whole-hypothalamus samples. Overall, these data indicate that a substantial proportion of $\alpha$-tanycytes have been lost in the hypothalamus of Gnasxl-deficient adult mice.

Two major functions of $\alpha$-tanycytes have been characterised. With their processes these cells bridge the ependymal border of the $3^{\text {rd }}$ ventricle with the Arc, VMH and $\mathrm{DMH}$ and are capable of transporting substrates from the cerebrospinal fluid into the hypothalamic parenchyma $[11,13,14]$. It was shown that $\alpha$-tanycytes express glucose transporters and are sensitive to changes in extracellular glucose concentration, thus implicating them as a functional component in the hypothalamic network that regulates energy homeostasis [11, 13]. We did not detect changes in glial transporter gene expression in our RNA samples from whole hypothalami, which could be due to compensatory up-regulation in the remaining cells. However, at this stage we cannot exclude disturbances in localised nutrient or neurotransmitter transport or availability.

Recent reports have shown that tanycytes also possess neural progenitor cell functions [13, 21, 23]. Both, $\beta$ - as well as $\alpha$-tanycytes can proliferate and generate neurons 
and to some extent glial cells, although $\beta$-tanycytes appear to exert this function mainly during juvenile stages and show less proliferation during adulthood $[25,26]$. By contrast, $\alpha$-tanycytes were shown to self-renew and give rise to neurons and glial cells at adult stages, and this process was specifically stimulated by fibroblast growth factors (FGFs) and insulin-like growth factor 1 (IGF-1) $[24,27,28]$. Some $\alpha$-tanycytes co-express Gfap and the neural stem cell transcription factor Sox2 [28], which showed a trend towards reduced expression levels in our RNAseq analysis of Gnasxl KO samples (Additional file 1). For another marker of neural progenitor cells, the intermediate filament protein Nestin, insufficient sequence reads were obtained in the RNAseq analysis, most likely due to low Nestin RNA levels, but it was detectable histologically in the ependymal layer. Nestin protein expression appeared to be reduced specifically at the level of $\alpha$-tanycytes, similar to Vimentin and Gfap. This finding further supports the view that the $\alpha$ tanycyte-associated neural progenitor cell population has been impacted. However, a functional assessment of hypothalamic stem cells in Gnasxl deficient mice and any consequences of a partial loss of $\alpha$-tanycytes on neural cell maintenance in the hypothalamus remain to be determined. Taking into account the nutritional programming effects discussed above, it can also not be excluded that postnatal undernutrition, either dietinduced [8-10] or as described in our genetic model $[38,39]$, results in additional changes in the hypothalamic network that regulates energy homeostasis. Future investigations will be aimed at assessing cell proliferation and potential changes in the proportions of specific neuron types in the hypothalamus, for example NPY and POMC neurons in the Arc.

The direct function of Gnasxl on a cellular and molecular level in neurons of the brain remains currently unclear. Although the $\mathrm{XL} \alpha_{\mathrm{s}}$ protein has been shown to couple activated receptors to the cAMP signalling pathway in cell lines [33] and can also mediate $\mathrm{G}_{\mathrm{q} / 11}$-like IP3 signalling in renal proximal tubules [32], the physiological relevant receptors in the brain have not yet been identified. Lack of $\mathrm{XL} \alpha_{\mathrm{s}}$ leads to severe postnatal undernutrition and failure to grow, which is associated with a high pre-weaning mortality [38]. KO mice that survive into adulthood show increased SNS activity, which causes an elevated basic metabolic rate, body temperature and cardiovascular parameters [37, 39]. Taking into account the data presented here, it appears now likely that the phenotype of Gnasx $l^{\mathrm{m}+/ \mathrm{p}-}$ mice that survive into adulthood needs to be considered as a composite of direct effects related to lack of $\mathrm{XL} \alpha_{\mathrm{s}}$ signalling in neurons, as well as adaptions in the hypothalamic neural network, including astrocytes and $\alpha$-tanycytes, which occur in response to the severe postnatal undernutrition of the mutant mice. The RNAseq data generated as part of this work will be a useful resource for comparisons with other nutritional programming studies, as well as for further investigations into the functions of Gnasxl in the hypothalamus. Additionally, the Gnasxl $\mathrm{KO}$ mouse model could have relevance to human in both aspects: in the context of rare genetic neonatal growth disorders that are associated with loss of GNASXL at chromosome 20q13 [51-55], and as a model to investigate the consequences of neonatal undernutrition on programming of energy homeostasis in human adult life [56].

\section{Conclusions}

Lean and hypermetabolic Gnasxl-deficient mice show reduced expression levels of the glial marker Gfap, as well as a reduction in astrocyte and $\alpha$-tanycyte numbers in the hypothalamus at adult stages. The findings that the changes are not present at postnatal stages when these glial cell types are completing their development and, furthermore, that Gnasxl is not expressed in these cell types, lead us to conclude that the hypothalamic changes are an adaptation or consequence of the severe neonatal undernutrition the $\mathrm{KO}$ mice experience. The adult phenotype of Gnasxl KO mice, which includes increased metabolic rate, reduced fat mass, increased glucose tolerance and insulin sensitivity, as well as elevated SNS activity, is reminiscent of rodent models of metabolic programming through postnatal undernutrition. The phenotype of adult Gnasxl-deficient mice, therefore, appears to be a combination of lack of $\mathrm{XL}_{\mathrm{s}}$ signalling in neurons, as well as adaptations in the hypothalamic network that regulates energy homeostasis and which includes astrocytes and $\alpha$-tanycytes.

\section{Methods}

\section{Animal care}

The Gnasxl mouse line [38] was maintained on a CD1 outbred background. Gnasxl-deficient offspring $\left(\right.$ Gnas $\left.x l^{\mathrm{m}+/ \mathrm{p}^{-}}\right)$, which lack $\mathrm{XL} \alpha_{\mathrm{s}}$ expression from the paternally inherited allele, was produced by mating CD1 females with Gnasxl mutation-carrying males. Mice were maintained in the animal facility of the University of Liverpool on a $12 \mathrm{~h}$ light - $12 \mathrm{~h}$ dark cycle and had unlimited access to water and standard chow diet. All animal work was approved by the Animal Welfare and Ethical Review Body of the University of Liverpool and carried out in accordance with the UK Animals (Scientific Procedures) Act 1986 (UK Home Office Project Licence PPL40/3577) and the directive 2010/63/EU for Europe.

\section{RNA seq and bioinformatics analyses}

Hypothalami were dissected from 6 Gnasx $^{\mathrm{m}+/ \mathrm{p}-}$ and $6 \mathrm{WT}$ littermates (six months old females). Hypothalamic total RNA was isolated using a Qiagen RNeasy Plus Mini kit. 
The quality of the RNA was confirmed on a Bioanalyzer 2100 (Agilent Technologies) with a RNA Integrity Number of 8.5 or higher for all samples. For cost effectiveness, but to retain biological sample variability, RNA from two Gnasx $^{\mathrm{m}+/ \mathrm{p}-}$ and two WT samples were pooled, respectively, to obtain a total of 3 Gnasx $l^{\mathrm{m}+/ \mathrm{p}-}$ and 3 WT RNA pools. Ribosomal RNA (rRNA) was removed from each pool using the Ribominus Eukaryote Kit for RNA-Seq (Life Technologies), and 95-98 \% depletion of rRNA was confirmed on a Bioanalyzer 2100. Before library preparation, the RNA sample concentrations were quantified on a Qubit (Life Technologies) using Ribogreen. For each pooled sample, $50 \mathrm{ng}$ of RNA was used for library preparation using the ScriptSeq v2 RNA-Seq Library Preparation Kit (Epicentre). Briefly, the RNA was first fragmented at $85^{\circ} \mathrm{C}$ for $5 \mathrm{~min}$, random hexamer cDNA synthesis primers with tagging sequences for Illumina sequencing slides were annealed and cDNA prepared using StarScript Reverse Transcriptase. cDNA was purified using the Agencourt AMPure XP system (BeckmanCoulter) and then amplified with ScriptSeq Index PCR Primers (Epicentre), in order to reduce representation bias during multiplex sequencing. Suitable size distribution of the library preparations were confirmed using the Bioanalyzer 2100. Pair-end sequencing ( 2 x 100 bp reads) of barcoded samples was carried out by the Centre for Genomic Research (University of Liverpool, UK) using an Illumina Genome Analyzer IIx (GAIIx). The data were quality-controlled using a filter for CHASTITY $\geq$ 0.6 and demultiplexed using the Illumina CASAVA 1.8.2 pipeline. A further quality control analysis was then performed using FastQC (Babraham Bioinformatics). Both sense and anti-sense FASTQ files were mapped to the mm10 build of the M. musculus genome (UCSC genome browser) using TopHat 2.0.4., incorporating a repeatmask (RepeatMasker) [57]. Of the approximately 28 million reads per sample generated, $40 \%$ were mapped to the mouse genome. Data were then analysed using Cufflinks, Cuffcompare and Cuffdiff (version 2.0.2) with multiple hypotheses correction [58] to the GTF transcript annotation file provided by Illumina iGenomes, to examine differential expression between Gnasx $l^{\mathrm{m}+/ \mathrm{p}-}$ and WT samples. RNAseq data files have been deposited at Gene Expression Omnibus (GEO) database under accession number GSE75878 (see also Additional file 1). Functional enrichment analysis was performed in the DAVID bioinformatics resources [59] using the whole mouse genome as background and default parameters. Full results, including $p$-values after correcting for multiple hypotheses testing, are available in Additional file 2.

\section{qRT-PCR}

Quantitative RT-PCR was carried out on the same three pooled WT and three pooled Gnasx $l^{\mathrm{m}+/ \mathrm{p}-}$ total RNA samples that had been used for the RNAseq experiments.
cDNA was synthesised using MMLV Reverse Transcriptase (Life Technologies) and real-time PCR was performed using the Brilliant II SYBR Green QPCR Master Mix with Low Rox (Agilent Technologies) on an Applied Biosystems 7500 Fast Real-Time PCR System. Relative quantification was calculated through normalisation to two housekeeping genes, $\beta$-Actin (Actb) and Cyclophilin A (Ppia), using the Applied Biosystems 7500 software. Primer efficiencies were evaluated on a five-fold dilution series of cDNA and only primer pairs with 93$107 \%$ efficiency and a $R^{2}>0.98$ were used for relative quantification. The following primer pairs were used: $A c t b$ : For 5'-GCTTCTTTGCAGCTCCTTCGT-3', Rev 5'ATATCGTCATCCATGGCGAAC-3'; Ppia: For 5'CAAATGCTGGACCAAACACAA-3', Rev 5'-GCCATCCAGCCATTCAGTCT-3'; Gfap: For 5'ACCAGCTTACGGCCAACAGT-3', Rev 5'-CCGAGGTCCTGTG CAAAGTT-3'. Glial solute transporter gene expression was assessed separately on a Biorad CFX Connect instrument using iTaq Universal SYBR Green Supermix reagents (Bio-Rad Laboratories). Hypothalamic RNA from six adult WT and six Gnasx $l^{m+/ p-}$ littermates was used. Target gene data were normalised to two housekeeping genes, $\beta$-Actin $(A c t b)$ and Beta-2-microglobulin $(B 2 m)$, and relative expression was calculated via the $\Delta \mathrm{Ct}$ method. The following primer pairs were used: Actb: For 5'-GGCTCCTAGCACCATGAAGATC-3', Rev 5'ACATCTGCTGGAAGGTGGACA-3'; B2m: For 5'-ATTCACCCCCACTGAGACTG-3', Rev 5'-GTCTCGATCCCAGTAGACGG-3'; Slc2a1: For 5'-CTCACCACGCT TTGGTCTCT-3', Rev 5'-CCCAGTTTGGAGAAGCCCAT-3'; Slc1a2: For 5'-ATCACTGCTCTGGGAACTGC3', Rev 5'-ACGAATCTGGTCACACGCTT; Slc1a3: For 5'GAGAGATTGCAGCAAGGGGT-3', Rev 5'-ATACGGTC GGAGGGCAAATC-3'.

\section{Immunohistochemistry}

For IHC, transcardiac perfusion was carried out on $5 \mathrm{WT}$ and 5 Gnasx $^{\mathrm{m}+/ \mathrm{p}-}$ (KO) littermates (2-4 months old females), brain tissues were further fixed overnight in $4 \%$ PFA/PBS and then dehydrated in $30 \%$ sucrose/PBS. Postnatal brains were fixed without perfusion. $12 \mu \mathrm{m}$ brain sections were prepared on a Leica CM 1950 cryostat. Sections were treated for antigen retrieval by submerging slides in $10 \mathrm{mM}$ sodium citrate at $60{ }^{\circ} \mathrm{C}$ for $2 \mathrm{~min}$ and endogenous peroxidase activity was quenched by submerging section in methanol/0.3 \% $\mathrm{H}_{2} \mathrm{O}_{2}$ for $5 \mathrm{~min}$. Sections were blocked with $\mathrm{PBS} / 10 \%$ goat serum $/ 0.25 \%$ Triton-X100, incubated overnight with rabbit polyclonal anti-Gfap (Dako, Z0334, 1:3000) or mouse monoclonal anti-Nestin (BD Pharmingen, 556309, 1:100) antibody and developed using the VECTASTAIN Elite ABC Kit (Vector Laboratories) with 3,3'-diaminobenzidine substrate. Stained sections were dehydrated, permanently mounted in Eukitt (Fluka 
Analyticals) and imaged on a Leica microscope and LAS software (Leica Microsystems). Hypothalamic Gfap cell counts were obtained using ImageJ from images taken at $100 \times$ magnification, whereby the $3^{\text {rd }}$ ventricle was positioned at the centre of the image. All areas along the dorso-ventral axis of the hypothalamic ventricle were included at equal left-right distance from the midline. Sections covered the rostro-caudal axis of the hypothalamus corresponding to the mouse brain atlas plates 36-50 (Bregma $-0.58 \mathrm{~mm}$ to $-2.30 \mathrm{~mm}$ ) [60], including the PVH, ME, Arc, VMH, DMH and PH. Images from WT and KO littermates that matched to the same atlas plate were paired and processed for comparative glial cell counts. In total, 22 pairs of sections from the $5 \mathrm{WT} / 5 \mathrm{KO}$ adult brains were included. For postnatal day 15, Gfap cell numbers were established from 33 pairs of sections from 3 WT/3 KO littermates from two litters. Images of adult hippocampus Gfap expression were taken from the same series of stained sections as the hypothalamus images, covering the mouse brain atlas plates 41-49 (Bregma -1.22 to $-2.18 \mathrm{~mm}$ ) [60]. In total, 15 pairs of sections from $5 \mathrm{WT} / 5 \mathrm{KO}$ mice were analysed. For immunofluorescence, the same anti-GFAP and a chicken anti-Vimentin antibody (Millipore, AB5733, 1:4000) were used, combined with Alexa Fluor 488 donkey anti rabbit (Life Technologies) and DyLight 594 donkey anti-chicken (Jackson ImmunoResearch Laboratories) secondary antibodies (1:2000).

\section{Statistical analyses}

Hypothalamic cell counts and qRT-PCR data were analysed by Student's independent $t$-test. RNAseq statistical analysis was undertaken as described above. $P$-values in Additional file 1 are derived from the corresponding $q$ values following multiple hypotheses correction. All graphs show data as means \pm SEM.

\section{Ethics approval}

All animal (mouse) work was approved by the Animal Welfare and Ethical Review Body of the University of Liverpool and carried out in accordance with the revised UK Animals (Scientific Procedures) Act 1986 (UK Home Office Project Licence PPL40/3577) and the directive 2010/63/EU for Europe.

\section{Consent for publication \\ Not applicable.}

\section{Availability of data and materials}

The RNAseq data have been deposited in the 'Gene Expression Omnibus (GEO)' database under accession number GSE75878. Further datasets supporting the conclusions of this article are included within the article and its additional files.

\section{Additional files}

Additional file 1: RNAseq list of gene expression changes in hypothalamus samples from Gnasx ${ }^{m+/ p-}$ mice versus WT littermates. Genes are listed by their log2(fold change) from most up-regulated to most down-regulated. (XLSX $5507 \mathrm{~kb})$

Additional file 2: Functional enrichment analysis using the DAVID bioinformatics resources. The whole mouse genome was used as background with default parameter settings. Full results, including $p$-values after correcting for multiple hypotheses testing, are shown. Statistically significant enriched categories amongst overexpressed genes related to the two broad categories of 'ribosomes/rRNA binding and translation' and 'mitochondria/electron transport chain'. Amongst the down-regulated genes no statistically significant categories were found. (XLSX $166 \mathrm{~kb}$ )

Additional file 3: Gfap expression in the hippocampus of adult Gnasxldeficient mice is not changed. $\mathrm{HC}$ for Gfap on coronal brain sections from WT (A, C, E) and KO (B, D, F) littermates at different rostro-caudal levels. In contrast to the hypothalamus, quantification of Gfap-expressing cells in the hippocampal areas did not show any significant difference $(\mathrm{WT}=769 \pm 16$ vs $\mathrm{KO}=746 \pm 19$ cells/section \pm sem, $p>0.05, t$-test, $n=15$ matched pairs of sections from $5 \mathrm{WT}$ and $5 \mathrm{KO}$ mice). Scale bar $=100 \mu \mathrm{m}$. (PDF $731 \mathrm{~kb}$ )

Additional file 4: Gnas $x$ l is not expressed in the ependymal layer, but the parenchyma of the hypothalamus. $\mathrm{HC}(\mathbf{A})$ for $X \mathrm{La}_{\mathrm{s}}$ (green) in the postnatal day 1 (P1) hypothalamus and in situ hybridisation for Gnasxl at P4 (B) show scattered positive cells in the DMH, but not in the ependymal layer (ep) of the $3^{\text {rd }}$ ventricle (DAPI nuclear counterstain). (C) Similarly, IHC for $\mathrm{XLa}_{\mathrm{s}}$ in the adult hypothalamus marks neurons in the parenchyma, but no expression is found in ependymal cells (arrow). XLa is a membraneassociated protein detectable in neurites, but no tanycyte extensions are stained. Arc $=$ arcuate nucleus, $\mathrm{DMH}=$ dorsomedial nucleus, $\mathrm{ME}=$ median eminence. Scale bar $=100 \mu \mathrm{m}$. (PDF $619 \mathrm{~kb}$ )

Additional file 5: Expression levels of glial solute transporter genes in hypothalami of adult Gnasx $\left.\right|^{m+/ p-}$ mice. qRT-PCR analyses did not detect any significant changes in expression levels of the glucose transporter S/c2a 1 (Glut1) or the excitatory amino acid transporters S/cla2 (G/t1) and S/cla3 (Glast1). (N=6 WT and $6 \mathrm{KO}$ samples; $t$-test, $p>0.05$ n.s.). (PDF 273 kb)

\section{Abbreviations}

Arc: arcuate nucleus; DMH: dorsomedial hypothalamic nucleus; Gas: G-protein a-stimulatory subunit; GEO: gene expression omnibus; ME: median eminence; IHC: immunohistochemistry; KO: knock-out; NPY: neuropeptide Y; PH: posterior hypothalamic area; POMC: pro-opiomelanocortin; PVH: paraventricular hypothalamic nucleus; rRNA: ribosomal RNA; SCN: suprachiasmatic nucleus; SNS: sympathetic nervous sysem; VMH: ventromedial hypothalamic nucleus; WT: wild-type; XLas: extra-large G-protein a-subunit.

\section{Competing interests}

The authors declare that they have no competing interests.

\section{Authors' contributions}

APH carried out the RNAseq and some qRT-PCR experiments, undertook the bioinformatics analyses and contributed to the IHC experiments. SQW, MP, $\mathrm{K}, \mathrm{NSH}$ and PT undertook IHC experiments and contributed to the GRT-PCR experiments. JPdM and AP conceived and designed the study and AP did the statistical analyses and wrote the manuscript. All authors read and approved the final manuscript.

\section{Acknowledgements}

We would like to thank the staff of the Biomedical Services Unit of the University of Liverpool for their excellent animal facility support.

\section{Funding}

This study was supported by a Biotechnology and Biological Sciences Research Council (BBSRC) studentship (BBD5265291) to A.P.H. and by the Wellcome Trust 
studentship programme in Cellular and Molecular Physiology at the University of Liverpool for S.Q.W. (102378/Z/13/Z) and M.P. (099795/Z/12/Z).

\section{Received: 21 December 2015 Accepted: 8 April 2016 Published online: 14 April 2016}

\section{References}

1. Kooijman S, van den Heuvel JK, Rensen PC. Neuronal Control of Brown Fat Activity. Trends Endocrinol Metab. 2015;26:657-68.

2. Morrison SF, Madden CJ, Tupone D. Central neural regulation of brown adipose tissue thermogenesis and energy expenditure. Cell Metab. 2014;19:741-56.

3. Sohn JW, Elmquist JK, Williams KW. Neuronal circuits that regulate feeding behavior and metabolism. Trends Neurosci. 2013:36:504-12.

4. Bouret SG, Simerly RB. Development of leptin-sensitive circuits. J Neuroendocrinol. 2007;19:575-82.

5. Coupe B, Bouret SG. Development of the hypothalamic melanocortin system. Front Endocrinol (Lausanne). 2013:4:38.

6. Elson AE, Simerly RB. Developmental specification of metabolic circuitry. Front Neuroendocrinol. 2015;39:38-51.

7. Dearden L, Ozanne SE. Early life origins of metabolic disease: Developmental programming of hypothalamic pathways controlling energy homeostasis. Front Neuroendocrinol. 2015:39:3-16.

8. Cripps RL, Martin-Gronert MS, Archer ZA, Hales CN, Mercer JG, Ozanne SE. Programming of hypothalamic neuropeptide gene expression in rats by maternal dietary protein content during pregnancy and lactation. Clin Sci (Lond). 2009;117:85-93.

9. Delahaye F, Lukaszewski MA, Wattez JS, Cisse O, Dutriez-Casteloot I, Fajardy I, et al. Maternal perinatal undernutrition programs a "brown-like" phenotype of gonadal white fat in male rat at weaning. Am J Physiol Regul Integr Comp Physiol. 2010;299:R101-10.

10. Stocker CJ, Wargent ET, Martin-Gronert MS, Cripps RL, O'Dowd JF, Zaibi MS, et al. Leanness in postnatally nutritionally programmed rats is associated with increased sensitivity to leptin and a melanocortin receptor agonist and decreased sensitivity to neuropeptide Y. Int J Obes (Lond). 2012;36:1040-6.

11. Argente-Arizon P, Freire-Regatillo A, Argente J, Chowen JA. Role of non-neuronal cells in body weight and appetite control. Front Endocrinol (Lausanne). 2015;6:42.

12. Balland E, Dam J, Langlet F, Caron E, Steculorum S, Messina A, et al. Hypothalamic tanycytes are an ERK-gated conduit for leptin into the brain. Cell Metab. 2014;19:293-301.

13. Bolborea M, Dale N. Hypothalamic tanycytes: potential roles in the control of feeding and energy balance. Trends Neurosci. 2013;36:91-100.

14. Rodriguez EM, Blazquez JL, Pastor FE, Pelaez B, Pena P, Peruzzo B, et al. Hypothalamic tanycytes: a key component of brain-endocrine interaction. Int Rev Cytol. 2005;247:89-164.

15. Berkseth KE, Guyenet SJ, Melhorn SJ, Lee D, Thaler JP, Schur EA, et al. Hypothalamic gliosis associated with high-fat diet feeding is reversible in mice: a combined immunohistochemical and magnetic resonance imaging study. Endocrinology. 2014;155:2858-67.

16. Buckman LB, Thompson MM, Moreno HN, Ellacott KL. Regional astrogliosis in the mouse hypothalamus in response to obesity. J Comp Neurol. 2013;521:1322-33.

17. Fuente-Martin E, Garcia-Caceres C, Granado M, de Ceballos ML, SanchezGarrido MA, Sarman B, et al. Leptin regulates glutamate and glucose transporters in hypothalamic astrocytes. J Clin Invest. 2012;122:3900-13.

18. Horvath TL, Sarman B, Garcia-Caceres C, Enriori PJ, Sotonyi P, Shanabrough $M$, et al. Synaptic input organization of the melanocortin system predicts diet-induced hypothalamic reactive gliosis and obesity. Proc Natl Acad Sci U S A. 2010;107:14875-80.

19. Lemus MB, Bayliss JA, Lockie SH, Santos W, Reichenbach A, Stark R, et al. A stereological analysis of NPY, POMC, Orexin, GFAP astrocyte, and Iba1 microglia cell number and volume in diet-induced obese male mice. Endocrinology. 2015;156:1701-13.

20. Thaler JP, Yi CX, Schur EA, Guyenet SJ, Hwang BH, Dietrich MO, et al. Obesity is associated with hypothalamic injury in rodents and humans. J Clin Invest. 2012;122:153-62.

21. Maggi R, Zasso J, Conti L. Neurodevelopmental origin and adult neurogenesis of the neuroendocrine hypothalamus. Front Cell Neurosci. 2014;8:440.

22. Migaud M, Batailler M, Segura S, Duittoz A, Franceschini I, Pillon D. Emerging new sites for adult neurogenesis in the mammalian brain: a comparative study between the hypothalamus and the classical neurogenic zones. Eur J Neurosci. 2010;32:2042-52
23. Sousa-Ferreira $L$, de Almeida LP, Cavadas C. Role of hypothalamic neurogenesis in feeding regulation. Trends Endocrinol Metab. 2014;25:80-8.

24. Xu Y, Tamamaki N, Noda T, Kimura K, Itokazu Y, Matsumoto N, et al. Neurogenesis in the ependymal layer of the adult rat 3rd ventricle. Exp Neurol. 2005;192:251-64.

25. Haan N, Goodman T, Najdi-Samiei A, Stratford CM, Rice R, El Agha E, et al. Fgf10expressing tanycytes add new neurons to the appetite/energy-balance regulating centers of the postnatal and adult hypothalamus. J Neurosci. 2013;33:6170-80.

26. Lee DA, Bedont JL, Pak T, Wang H, Song J, Miranda-Angulo A, et al. Tanycytes of the hypothalamic median eminence form a diet-responsive neurogenic niche. Nat Neurosci. 2012;15:700-2.

27. Perez-Martin M, Cifuentes M, Grondona JM, Lopez-Avalos MD, GomezPinedo U, Garcia-Verdugo JM, et al. IGF-I stimulates neurogenesis in the hypothalamus of adult rats. Eur J Neurosci. 2010;31:1533-48.

28. Robins SC, Stewart I, McNay DE, Taylor V, Giachino C, Goetz M, et al. aTanycytes of the adult hypothalamic third ventricle include distinct populations of FGF-responsive neural progenitors. Nat Commun. 2013;4:2049.

29. Weinstein LS. Role of $\mathrm{G}(\mathrm{S}) \mathrm{a}$ in central regulation of energy and glucose metabolism. Horm Metab Res. 2014;46:841-4.

30. Peters J, Williamson CM. Control of imprinting at the Gnas cluster. Epigenetics. 2007;2:207-13.

31. Plagge A, Kelsey G, Germain-Lee EL. Physiological functions of the imprinted Gnas locus and its protein variants Gas and XLas in human and mouse. J Endocrinol. 2008;196:193-214.

32. He Q, Zhu Y, Corbin BA, Plagge A, Bastepe M. The G protein a subunit variant XLas promotes inositol 1,4,5-trisphosphate signaling and mediates the renal actions of parathyroid hormone in vivo. Sci Signal. 2015;8:ra84.

33. Kaya Al, Ugur O, Oner SS, Bastepe M, Onaran HO. Coupling of $\beta 2-$ adrenoceptors to XLas and Gas: a new insight into ligand-induced G protein activation. J Pharmacol Exp Ther. 2009;329:350-9.

34. Peters $\mathrm{J}$. The role of genomic imprinting in biology and disease: an expanding view. Nat Rev Genet. 2014;15:517-30.

35. Chen M, Berger A, Kablan A, Zhang J, Gavrilova O, Weinstein LS. Gsa deficiency in the paraventricular nucleus of the hypothalamus partially contributes to obesity associated with Gsa mutations. Endocrinology. 2012;153:4256-65.

36. Chen M, Wang J, Dickerson KE, Kelleher J, Xie T, Gupta D, et al. Central nervous system imprinting of the $\mathrm{G}$ protein $\mathrm{G}(\mathrm{s}) \mathrm{a}$ and its role in metabolic regulation. Cell Metab. 2009;9:548-55.

37. Nunn N, Feetham CH, Martin J, Barrett-Jolley R, Plagge A. Elevated blood pressure, heart rate and body temperature in mice lacking the XLas protein of the Gnas locus is due to increased sympathetic tone. Exp Physiol. 2013;98:1432-45.

38. Plagge A, Gordon E, Dean W, Boiani R, Cinti S, Peters J, et al. The imprinted signaling protein $\mathrm{XLas}$ is required for postnatal adaptation to feeding. Nat Genet. 2004;36:818-26.

39. Xie T, Plagge A, Gavrilova O, Pack S, Jou W, Lai EW, et al. The Alternative Stimulatory G Protein a-Subunit XLas Is a Critical Regulator of Energy and Glucose Metabolism and Sympathetic Nerve Activity in Adult Mice. J Biol Chem. 2006;281:18989-99.

40. Krechowec SO, Burton KL, Newlaczyl AU, Nunn N, Vlatkovic N, Plagge A. Postnatal Changes in the Expression Pattern of the Imprinted Signalling Protein XLas Underlie the Changing Phenotype of Deficient Mice. PLoS One. 2012;7, e29753.

41. de Magalhaes JP, Finch CE, Janssens G. Next-generation sequencing in aging research: emerging applications, problems, pitfalls and possible solutions. Ageing Res Rev. 2010;9:315-23.

42. Wood SH, Craig T, Li Y, Merry B, de Magalhaes JP. Whole transcriptome sequencing of the aging rat brain reveals dynamic RNA changes in the dark matter of the genome. Age (Dordr). 2013;35:763-76.

43. Freeman MR. Specification and morphogenesis of astrocytes. Science. 2010;330: 774-8.

44. Berger UV, Hediger MA. Differential distribution of the glutamate transporters GLT-1 and GLAST in tanycytes of the third ventricle. J Comp Neurol. 2001;433:101-14.

45. Peruzzo B, Pastor FE, Blazquez JL, Schobitz K, Pelaez B, Amat P, et al. A second look at the barriers of the medial basal hypothalamus. Exp Brain Res. 2000;132:10-26.

46. Miranda-Angulo AL, Byerly MS, Mesa J, Wang H, Blackshaw S. Rax regulates hypothalamic tanycyte differentiation and barrier function in mice. J Comp Neurol. 2014;522:876-99.

47. Salvatierra J, Lee DA, Zibetti C, Duran-Moreno M, Yoo S, Newman EA, et al. The LIM homeodomain factor Lhx2 is required for hypothalamic tanycyte specification and differentiation. J Neurosci. 2014;34:16809-20. 
48. Rocha ML, Fernandes PP, Lotufo BM, Manhaes AC, Barradas PC, Tenorio F. Undernutrition during early life alters neuropeptide $Y$ distribution along the arcuate/paraventricular pathway. Neuroscience. 2014;256:379-91.

49. Aguilar-Roblero R, Salazar-Juarez A, Rojas-Castaneda J, Escobar C, Cintra L. Organization of circadian rhythmicity and suprachiasmatic nuclei in malnourished rats. Am J Physiol. 1997;273:R1321-31.

50. Mendonca JE, Vilela MC, Bittencourt H, Lapa RM, Oliveira FG, Alessio ML, et al. GFAP expression in astrocytes of suprachiasmatic nucleus and medial preoptic area are differentially affected by malnutrition during rat brain development. Nutr Neurosci. 2004;7:223-34.

51. Balasubramanian M, Atack E, Smith K, Parker MJ. A novel de novo 20q13.32q13.33 deletion in a 2-year-old child with poor growth, feeding difficulties and low bone mass. J Hum Genet. 2015;60:313-7.

52. Chudoba I, Franke Y, Senger G, Sauerbrei G, Demuth S, Beensen V, et al. Maternal UPD 20 in a hyperactive child with severe growth retardation. Eur J Hum Genet. 1999;7:533-40.

53. Genevieve D, Sanlaville D, Faivre L, Kottler ML, Jambou M, Gosset P, et al. Paternal deletion of the GNAS imprinted locus (including Gnasxl) in two girls presenting with severe pre- and post-natal growth retardation and intractable feeding difficulties. Eur J Hum Genet. 2005;13:1033-9.

54. Mulchandani S, Bhoj EJ, Luo M, Powell-Hamilton N, Jenny K, Gripp KW, et al. Maternal uniparental disomy of chromosome 20: a novel imprinting disorder of growth failure. Genet Med. 2016;18:309-15.

55. Richard N, Molin A, Coudray N, Rault-Guillaume P, Juppner H, Kottler ML. Paternal GNAS mutations lead to severe intrauterine growth retardation (IUGR) and provide evidence for a role of XLas in fetal development. J Clin Endocrinol Metab. 2013;98:E1549-56.

56. Druet C, Ong KK. Early childhood predictors of adult body composition. Best Pract Res Clin Endocrinol Metab. 2008;22:489-502.

57. Tarailo-Graovac M, Chen N. Using RepeatMasker to identify repetitive elements in genomic sequences. Curr Protoc Bioinformatics. 2009. Chapter 4:Unit 4 10; doi: 10.1002/0471250953.bi0410s25.

58. Trapnell C, Williams BA, Pertea G, Mortazavi A, Kwan G, van Baren MJ, et al. Transcript assembly and quantification by RNA-Seq reveals unannotated transcripts and isoform switching during cell differentiation. Nat Biotechnol. 2010;28:511-5.

59. da Huang W, Sherman BT, Lempicki RA. Systematic and integrative analysis of large gene lists using DAVID bioinformatics resources. Nat Protoc. 2009;4:44-57.

60. Paxinos G, Franklin KBJ. The mouse brain in stereotaxic coordinates. 2nd ed. San Diego: Academic; 2001.

\section{Submit your next manuscript to BioMed Central and we will help you at every step:}

- We accept pre-submission inquiries

- Our selector tool helps you to find the most relevant journal

- We provide round the clock customer support

- Convenient online submission

- Thorough peer review

- Inclusion in PubMed and all major indexing services

- Maximum visibility for your research

Submit your manuscript at www.biomedcentral.com/submit 